\title{
LHCb results with vector bosons
}

\author{
Donatella Lucchesi* ${ }^{\dagger}$ \\ University and INFN of Padova \\ E-mail: donatella.lucchesiepd.infn.it
}

Measurements of electroweak bosons production provide an important test of the Standard Model at the LHC energies and allow the partonic content of the proton to be constrained. The LHCb forward acceptance is suited for measurements complementary to the other LHC experiments. $W$ and $Z$ bosons are reconstructed in $e$ and $\mu$ leptonic final states using data samples collected at energies in the center of mass frame of $\sqrt{s}=7,8,13 \mathrm{TeV}$ corresponding to integrated luminosities of $1,2,0.29 \mathrm{fb}^{-1}$ respectively. Results on $W+b / c$ quark and on $W / Z+$ jets are also presented.

38th International Conference on High Energy Physics 3-10 August 2016

Chicago, USA

* Speaker.

${ }^{\dagger}$ On behalf of the LHCb Collaboration 


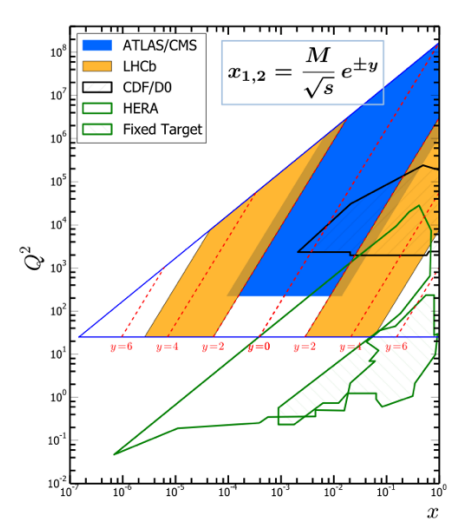

Figure 1: The $\left(x, Q^{2}\right)$ plane and where it is covered by each experiment.

\section{Introduction}

Theoretical predictions of electroweak bosons production are available at next-to-next-toleading order (NNLO) in perturbative quantum chromodynamics and rely on the parameterizations of the momentum fraction, Bjorken-x $(x)$, of the partons inside the colliding particles. While the partonic cross-sections are well understood, the knowledge of the Parton Density Functions (PDF) which parameterize the behavior of the partons within the colliding protons is a source of additional uncertainty. LHCb probes a unique region of $\left(x, Q^{2}\right)$ space, with $\mathrm{Q}$ the energy transfered in the interaction, at LHC. The $\left(x, Q^{2}\right)$ coverage of the LHCb detector [1] at $\sqrt{s}=13 \mathrm{TeV}$, compared to the other experiments is shown in figure 1 .

\section{Inclusive $Z$ and $W$ production cross section measurements}

LHCb results on inclusive $Z$ and $W$ production are sensitive to effects at low and high values of $x$ due to the longitudinal boost required for the bosons to be produced in the forward region. The $Z$ boson transverse momentum $\left(p_{T}\right)$ and $\phi^{*}$ [2] distributions together with the lepton pseudorapidity, $\eta_{l}$, distribution in the $W$ decay can also be used to test Monte Carlo modeling of additional higherorder radiation that arises from quantum chromodynamics (QCD).

\section{$2.1 Z \rightarrow l l$ production at $\sqrt{s}=13 \mathrm{TeV}$}

The $Z \rightarrow \mu \mu$ candidates are identified by requiring two reconstructed muons with opposite charge, $p_{T}>20 \mathrm{GeV} / \mathrm{c}, 2 \leq \eta \leq 4.5$ and invariant mass $60<M(\mu \mu)<120 \mathrm{GeV} / \mathrm{c}^{2}$. The major background contributions come from semileptonic decays of heavy flavour hadrons and QCD events where kaons or pions are falsely identified as muons. Data drive methods are used to estimate the fraction of these events in the selected sample while Monte Carlo simulation gives the $Z \rightarrow \tau \tau$, top and di-boson contributions. The $Z \rightarrow e e$ events are reconstructed using the same fiducial acceptance. Background due to particle misidentification is estimated using a data sample of same-sign electron pairs and contribution from heavy flavour hadrons and $Z \rightarrow \tau \tau$ is found to be negligible in this case. The inclusive $Z$ boson cross-section for decays to a dilepton final state is measured [3] to be 


$$
\begin{aligned}
& \sigma_{Z}^{\mu \mu}=198.0 \pm 0.9(\text { stat } .) \pm 4.7(\text { sys. }) \pm 7.7(\text { lumi }) p b \\
& \left.\sigma_{Z}^{e e}=190.2 \pm 1.7 \text { (stat. }\right) \pm 4.7(\text { sys. }) \pm 7.4(\text { lumi }) p b
\end{aligned}
$$

Taking the luminosity uncertainty to be fully correlated, the two measurements are consistent at the level of $1.1 \sigma$, and are linearly combined to give [3]

$$
\sigma_{Z}^{l l}=194.3 \pm 0.9(\text { stat } .) \pm 3.3(\text { sys. }) \pm 7.6(\text { lumi }) p b
$$

Figure 2 show the measured cross section compared to theory. The top three points correspond to predictions with different PDF sets. The bottom points correspond to the LHCb measurements in the dielectron and dimuon final states and their average. In addition the $Z$ boson production cross

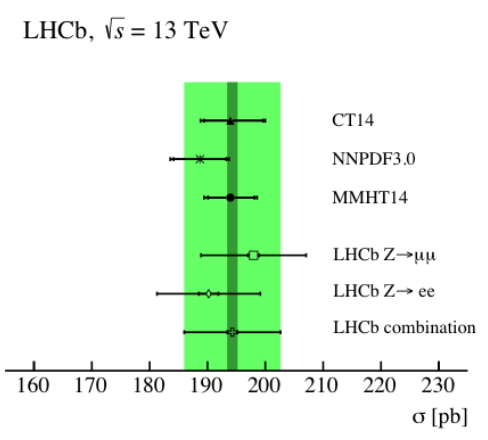

Figure 2: The $Z$ cross-section compared between theory and data. The bands correspond to the average of the dimuon and dielectron final states, with the inner band corresponding to the statistical uncertainty and the outer band corresponding to the total uncertainty.

section is measured in bins of $y_{Z}, \phi^{*}$, and, for the dimuon final state, in bins of the boson $p_{T}$ [3].

\section{$2.2 W \rightarrow l v$ production at $\sqrt{s}=8 \mathrm{TeV}$}

$W$ candidates are selected by requiring an isolated prompt muon or electron with $p_{T}>20 \mathrm{GeV}$ with $2 \leq \eta \leq 4.5$. The signal purity is obtained by fitting the $p_{T}$ distribution of the data sample in eight bins of lepton pseudorapidity for both charges, simultaneously, to the expected shapes for signal and background. The background contributions in the $W \rightarrow \mu v$ are the decay in flight of pions and kaons, the semi-leptonic decay of heavy flavour mesons, $Z \rightarrow \mu \mu$ events where one of the muons is not reconstructed in the LHCb acceptance and $Z \rightarrow \tau \tau$ events containing a single muon in the final state. The $W \rightarrow e v$ has significant background contribution from $Z \rightarrow e e$ with one electron in the LHCb acceptance, $Z \rightarrow \tau \tau$ and $W \rightarrow \tau \nu$ where the $\tau$ decays to a final state containing an electron. Prompt photon production in association with jets contributes in cases where the photon converts to an ee pair and only one electron is reconstructed and selected. The signal yield is corrected for losses due to reconstruction and selection efficiency, acceptance and final-state radiation. The $W^{+} / W^{-}$cross-section ratio and the production charge asymmetry of the $W$ boson are measured [4, 5], to test primarily the ratio of $u$ and $d$ quark densities. The lepton universality is quantified by computing a ratio of $W$ branching fractions using cross-sections determined in the range $2^{\prime} \eta^{l} \leq 3.5$ The results are compared to past measurements in figure 3 , the precision is seen to exceed previous individual determinations of the ratio and to be comparable to the combined LEP result. 


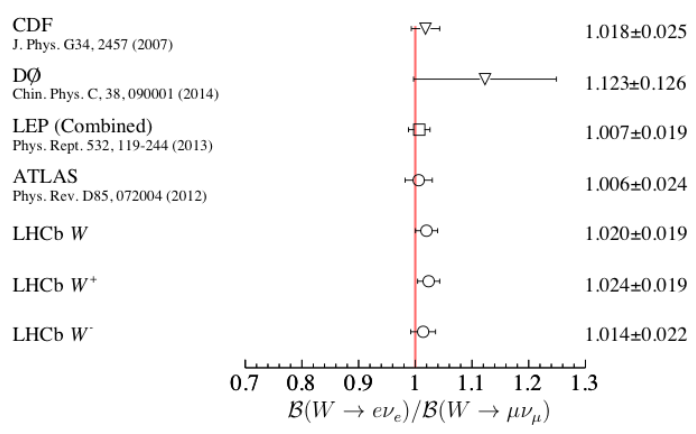

Figure 3: The ratio of branching fractions for the electron and muon final states determined for $W, W^{+}$and $W^{-}$is compared to hadron collider and LEP results with the theory represented by the red line.

\section{$2.3 W$ and $Z$ cross section ratios}

Ratios of the $W$ and $Z$ cross-sections provide precise tests of the standard model (SM) as the sensitivity to the PDF in the theoretical calculations is reduced and many of the experimental uncertainties cancel. In addition, the evolution of the cross-sections, and cross-section ratios, may be used to infer the existence of New Physics. LHCb has measured $W$ and $Z$ cross sections at $\sqrt{s}=7,8 \mathrm{TeV}$ using the muon and electron decay channel using the procedures described in the previous section. On the ratios of cross sections many sources of systematic uncertainties cancel or are reduced. The luminosity uncertainty completely cancels, as do the trigger efficiencies. The uncertainties on the tracking and muon identification efficiencies partially cancel in the ratios of $W$ and $Z$ boson cross sections, as do the uncertainties due to the proton beam energies.

The ratios of production cross section of $W^{+}$to $W^{-}, W^{ \pm}$to $Z$ and between $W^{+}, W^{-}$, and $Z$ at $\sqrt{s}=7,8 \mathrm{TeV}$ are shown in figure 4 .
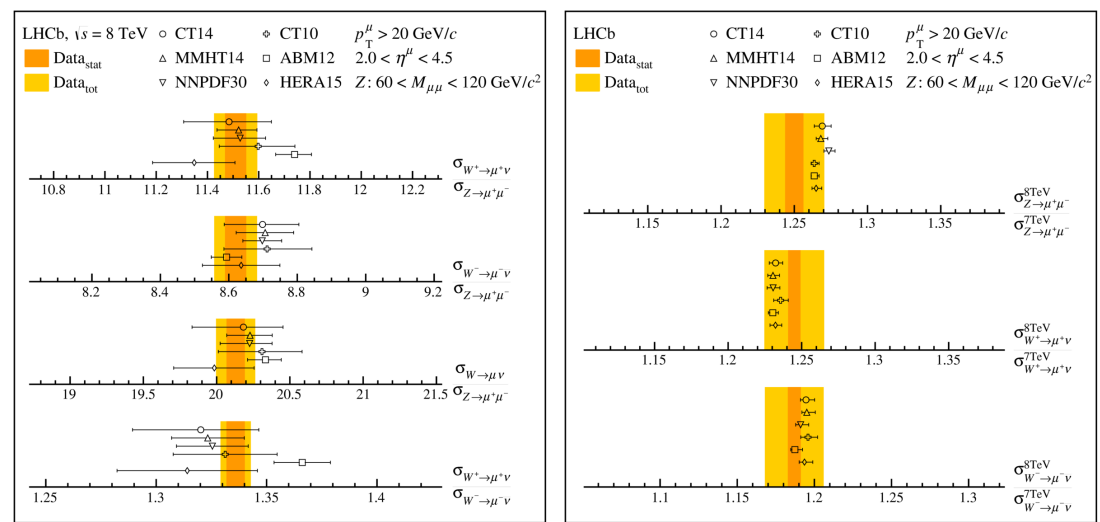

Figure 4: Left: summary of the $W$ and $Z$ cross-section ratios. Right: ratios of bosons cross sections at different centre-of-mass energies. Measurements, represented as bands, are compared to (markers) NNLO predictions with different parameterizations of the PDF.

\section{3. $W / Z$ plus jets cross section measurements}

Measurements of vector boson production in association with jets in the forward region can 
be used to test the SM and provide constraints on the PDF [6]. $W$ and $Z$ bosons are reconstructed with the same procedure previously described. In addition at least one jet per event with transverse momentum $p_{T}^{\text {jet }}>20 \mathrm{GeV}$ and pseudorapidity, $\eta^{\text {jet }}, 2.2<\eta^{j e t}<4.2$ is required. Jets are reconstructed using the anti- $k_{T}$ algorithm, with the $\mathrm{R}$ parameter set to 0.5 [7]. The jet is also required to be separated from the charged lepton(s) produced in the boson decay. The samples are selected in such a way they have the same fiducial acceptance. The main background contributions come from three processes, QCD multi-jet production, "fake jets" and electroweak process like $Z \rightarrow \tau \tau$ or $W \rightarrow \tau v$. The $W+$ jet yield is determined by performing an extended maximum likelihood fit to the distribution $p_{T}(\mu) / p_{T}\left(j_{\mu}\right)$, the muon transverse momentum divided by the transverse momentum of the $\mu$ - jet, reconstructed by replacing the muon with the jet that contains the muon after performing a jet reconstruction with relaxed jet selection requirements. This variable is a kind of muon isolation, providing strong discrimination between muons produced in electroweak processes, which tend to be isolated, and those produced in QCD processes, which are typically surrounded by additional particles. The background contribution to the $Z+$ jets sample is evaluated using both Monte Carlo and background enriched samples. Figure 5 summarizes the $W^{ \pm}+$jets and $Z+$ jets cross sections, the ratios of cross sections and the charge asymmetry of $W^{+}+$jets and $W^{-}+$jets compared to the theoretical predictions calculated using the aMC@NLO and Powheg generators.

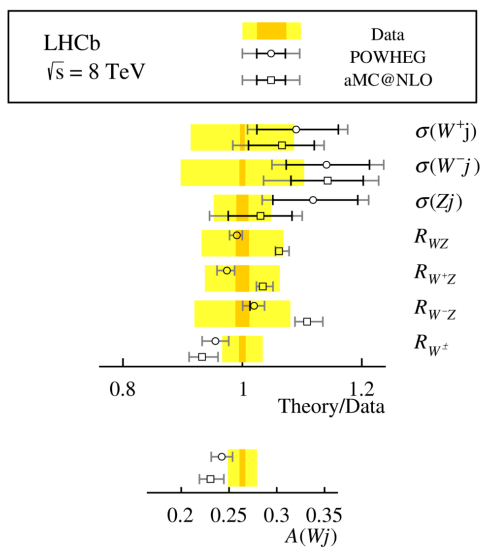

Figure 5: Summary of the measurements performed in the $W / Z$ plus jets sample. The measurements are shown as bands, while the theoretical predictions are presented as points. For the experimental measurements, the inner band represents the statistical uncertainty, while the outer band is the total uncertainty.

Of particular interest is the production of $W+b, c-$ jets [8]. The ratio of the $W+b-$ jets to $W+$ jets cross sections places constraints both on the intrinsic $b$ quark content of the proton and the probability of gluon splitting into $b \bar{b}$ pairs. The production of $W+c$-jets involves the $s$ quark therefore measuring the ratio of the $W+c$-jets to $W+$ jets production cross sections in the forward region provides important constraints on the $s$-quark PDF at momentum transfers of $Q^{2} 100 \mathrm{GeV}^{2}$ and $x 10^{-5}$. The $W$ boson and jets are reconstructed as previously described, the $b$ and $c$ jets are identified using the tagging algorithm described in [9] which exploits the presence of secondary vertices in the jets. The $p_{T}(\mu) / p_{T}\left(j_{\mu}\right)$ is fitted to determine the $W+$ jets yield and then in each bin of $p_{T}(\mu) / p_{T}\left(j_{\mu}\right)$ the two-dimensional tagger response distributions are fitted to determine the yields of $b$-tagged and $c$-tagged jets, which are used then to form the $p_{T}(\mu) / p_{T}\left(j_{\mu}\right)$ 
distributions for candidates with $b / c$-tagged jets. These distributions are fitted to determine the $W+b$ and $W+c$ yields by taking into account the background contribution coming from $Z+$ jets, $\mathrm{QCD}$, top production and $Z \rightarrow \tau \tau$. The measurement of $W+b, W+c$, and the $W+b / c$ asymmetry are performed at $\sqrt{s}=7$ and $8 \mathrm{TeV}$ as shown in figure 6 . The cross section ratio and

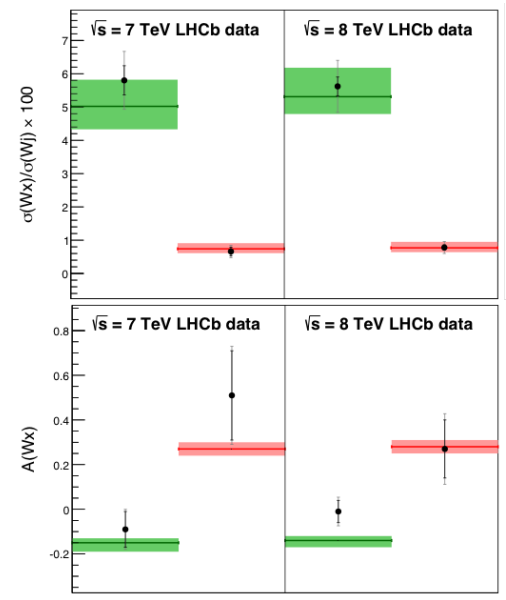

Figure 6: The first bin in each plot shows the $W+c$, the second one the $W+b$ results compared to SM predictions with the CT10 PDF set for $\sqrt{s}=7$ (left) and $\sqrt{s}=8$ (right). On the top figure the cross sections ratios measurements are shown while the bottom ones report the $W+b / c$ charge asymmetries.

the charge asymmetry for $W+b$ is consistent with theoretical calculations that assume no intrinsic $b$ quark content in the proton. The $W+c$ production is consistent with the expectations while the charge asymmetry is about $2 \sigma$ smaller than predicted and this could suggest a larger than expected contribution from scattering of strange quarks or a charge asymmetry between $s$ and $\bar{s}$ quarks in the proton.

\section{References}

[1] LHCb Coll., A. A. Alves Jr. et al., The LHCb detector at the LHC, JINST 3 (2008) S08005.

[2] A. Banfi et al., Optimisation of variables for studying dilepton transverse momentum distributions at hadron colliders, Eur. Phys. J. C71 (2011) 1600.

[3] LHCb Coll., Measurement of the forward Z boson production cross-section in pp collisions at $\sqrt{s}=13$ TeV, JHEP09 (2016) 136.

[4] LHCb Coll., Measurement of forward $\mathrm{W}$ and $\mathrm{Z}$ boson production in pp collisions at $\sqrt{s}=8 \mathrm{TeV}$, JHEP 01 (2016) 155.

[5] LHCb Coll., Measurement of forward $W \rightarrow e v$ production in pp collisions at $\sqrt{s}=8 \mathrm{TeV}$, JHEP 10 (2016) 030.

[6] LHCb Coll., Measurement of forward $W$ and $Z$ boson production in association with jets in proton-proton collisions at $\sqrt{s}=8 \mathrm{TeV}$, Journal of High Energy Physics, 2016(5), 1-23.

[7] LHCb Coll., Study of forward Z+jet production in pp collisions at $\sqrt{s}=8 \mathrm{TeV}$, JHEP01 (2014) 033

[8] LHCb Coll. Study of W boson production in association with beauty and charm, Phys. Rev. D 92, 052001 (2015)

[9] LHCb Coll., Identification of beauty and charm quark jets at LHCb, JINST 10 (2015) P06013. 